\title{
Using binaries containing giants to constrain theories of stellar and tidal evolution
}

\author{
A. Claret \\ Instituto de Astrofísica de Andalucía, CSIC, Apartado 3004, 18080 Granada, Spain \\ e-mail: claret@iaa.es
}

Received 20 February 2009 / Accepted 4 August 2009

\begin{abstract}
Aims. Investigations of stellar and tidal evolution of binary stars with giant components are rare. In this paper, we will investigate such features in three binary systems for which at least one component is a giant star. As some of these giants seem to be in the blue loop, it is an excellent opportunity to investigate the sensitivity of core overshooting on their location in the HR Diagram. We expect that these characteristics shall serve as an incentive to observers to investigate such kinds of binaries, increasing the accuracy of measurements and the number of systems to test the evolutionary models.

Methods. Prior to performing the study of the circularization and synchronization levels, an analysis of the capability of our stellar evolutionary models to reproduce the observed masses, radii and effective temperatures is carried out. Next, the differential equations of tidal evolution are integrated and the corresponding critical times are compared with the inferred age of the system and with the observed eccentricity and rotational velocities (when available).

Results. We have found good agreement between our stellar models and the astrophysical properties of $\eta$ And, V2291 Oph and SZ Cen by adopting a moderate core overshooting amount $\left(\alpha_{\mathrm{ov}}=0.20\right)$. Three mechanisms were used to try to explain the observed levels of circularization and synchronization: the hydrodynamical mechanism, turbulent dissipation and radiative damping. In the cases of $\eta$ And and SZ Cen, for which the rotational velocities are available, by assuming solid body rotation for both stars of each system we have found that the theoretical ratio between the rotational velocities $V_{\text {rota }} / V_{\text {rotB }}$ at the inferred ages are in good agreement with the observational ratios.
\end{abstract}

Key words. stars: binaries: general - stars: evolution - stars: rotation

\section{Introduction}

Tidal interactions in a binary system may lead to a final coalescence of both stars or, less drastically, to an equilibrium state, characterized by a circular coplanar orbit and with the rotational velocities synchronized with the orbital period. The evolutionary models, on the other hand, can provide in many cases a good approximation to the age of the system. By comparing this age with the critical times of circularization and synchronization computed by using current tidal theories, we can extract some information from the observed rotational velocities and eccentricity. As the time scales are strongly dependent on the absolute dimensions (mainly the radius), the best candidates for such a study are the double-lined eclipsing binaries with good determination of absolute dimensions. This kind of systematic investigation was already carried out by Claret \& Cunha (1997), for example. However, the sample adopted in that paper and others was constituted basically of Main-Sequence stars and the behaviour of more evolved components, concerning tidal interactions, was studied only in a few systems: $\lambda$ And, AY Cet and TZ For (Habets \& Zwaan 1989; Claret \& Giménez 1995). Only in the case of TZ For was it possible to determine the masses, radii and effective temperature with good accuracy.

Tidal evolution also can be investigated in binaries located in clusters since they can give information on the level of circularization of these coeval samples. One of the most important observations concerning binaries in clusters is the cut-off period (Mayor \& Mermilliod 1984), that is the period characterizing a transition between circular and eccentric orbits. This concept was recently revised (see for example Claret 2005; Meibom \& Mathieu 2005). Comparison between the cut-off periods (or their recently introduced equivalents) with those inferred from the different mechanisms of circularization is also a good tool to test current tidal theories.

In the present work we investigate the stellar and tidal evolution of three binary systems for which at least one component is a giant star. There are some advantages to the use of such rare systems: a) although the precision is not as high as in the case of the double-lined eclipsing binaries, the masses and radii of giants are determined with an acceptable accuracy; b) from the stellar evolution point of view, there is an extra advantage since the components are well separated and the models for single stars can be applied; c) as pointed out by Schröder, Pols \& Eggleton (1997), some of the $\zeta$ Aur giants are in the blue loop phase, which makes them an excellent test of the amount of core overshooting. The uncertainties involved in the analysis of our sample stars are admitedly quite large, if compared with those derived from double-lined eclipsing binaries. Therefore, the conclusions should be taken with caution. In order to illustrate the potential of the method in more accurate systems, we also introduce a double-lined eclipsing binary (SZ Cen) which presents moderately evolved stars with good determinations of the absolute dimensions.

The paper is organized as follows: first we introduce the main astrophysical parameters of our sample and the evolutionary stellar models which fit such properties. Next, the differential equations of tidal evolution are presented and some discussion on its derivation is also introduced, as well as the numerical 
Table 1. Astrophysical parameters for $\eta$ And, V2291 Oph, SZ Cen (solar, $\mathrm{K}$ units, $\mathrm{km} \mathrm{s}^{-1}$, days).

\begin{tabular}{llll}
\hline \hline & $\eta$ And & V2291 Oph & SZ Cen \\
\hline$M_{1}$ & $2.395 \pm 0.087$ & $3.86 \pm 0.15$ & $2.317 \pm 0.026$ \\
$M_{2}$ & $2.268 \pm 0.082$ & $2.95 \pm 0.09$ & $2.277 \pm 0.021$ \\
$\log \left(L_{1} / L_{\odot}\right)$ & $1.81 \pm 0.02$ & $2.73 \pm 0.08$ & $1.77 \pm 0.007$ \\
$\log \left(L_{2} / L_{\odot}\right)$ & $1.60 \pm 0.02$ & $2.07 \pm 0.08$ & $1.64 \pm 0.07$ \\
$\log T_{\text {eff1 }}$ & $3.703 \pm 0.007$ & $3.686 \pm 0.009$ & $3.875 \pm 0.017$ \\
$\log T_{\text {eff2 }}$ & $3.700 \pm 0.007$ & $4.041 \pm 0.019$ & $3.892 \pm 0.017$ \\
$R_{1}$ & 10.5 & $32.9 \pm 1.5$ & $4.554 \pm 0.032$ \\
$R_{2}$ & 8.5 & $3.0 \pm 0.2$ & $3.624 \pm 0.026$ \\
$e$ & $0.0034 \pm 0.0020$ & 0.311 & 0.0 \\
$P$ & 115.7 & 385 & 4.11 \\
$V_{\text {rotA }}$ & 9 & - & $60 \pm 5$ \\
$V_{\text {rotB }}$ & 9 & - & $44 \pm 4$ \\
Ref. & $1,2,3,6$ & 1,2 & 4,5 \\
\hline
\end{tabular}

References to Table 1: 1 Schröder et al. (1997); 2 Iwamoto \& Saio (1999); 3 Griffin (private comunication); 4 Andersen (1975); 5 Andersen (1991); 6 Massarotti et al. (2008).

method used to integrate them. Finally, once the stellar models are able to reproduce the observations, we use them to compute the critical times of circularization and synchronization and compare them to the observed rotational velocities (if available) and orbital eccentricities.

\section{Stellar models for $\boldsymbol{\eta}$ And, V2291 Oph and SZ Cen}

$\eta$ And (this star is also designed as HR 271, HD 5516 and HIP 4463) was discovered to be a spectroscopic binary by Campbell \& Wright (1900). Gordon (1946) obtained several spectrograms and determined its spectroscopic orbit. More recently Hummel et al. (1993) determined the orbit of the system by using optical interferometry. The masses of the primary and secondary inferred from these observations and from the spectroscopic studies by Gordon are $2.59 \pm 0.30 M_{\odot}$ and $2.34 \pm 0.22 M_{\odot}$.

The astrophysical parameters of $\eta$ And have been used to test the capability of evolutionary stellar models (Schröder et al. 1997; Iwamoto \& Saio 1999). In both works the primary seems to be in the core helium burning phase while the secondary is in the ascending giant branch. However, no studies on tidal effects were carried out in these papers. The masses used in such papers are somewhat different from those derived by Hummel et al. (1993). Here we adopted the masses from a recent spectroscopic orbital solution (Griffin, private communication). We will use the following values: $M_{1}=2.395 \pm 0.087 M_{\odot}$ and $M_{2}=2.268 \pm 0.082 M_{\odot}$, where $M_{1}$ and $M_{2}$ are the masses of the primary and secondary, respectively (see Table 1 ). On the other hand, Massarotti et al. (2008) presented rotational and radial velocities for 761 Hipparcos giants, including $\eta$ And. Considering the observational values of the eccentricity and the rotational velocities, it is possible to carry out an investigation of the tidal braking of this system, in addition to the traditional comparison between the observed astrophysical parameters (masses, radii, effective temperatures) and the theoretical predictions provided by the evolutionary models.

Prior to comparing the observed and theoretical levels of circularization and synchronization of $\eta$ And, V2291 Oph and SZ Cen, we need to check the capability of our stellar models to fit their observed properties. The basic properties of the code we use to generate the stellar models can be found in Claret (2004) and references given therein. For completeness, we enumerate below its main characteristics:

1. The convective core overshooting can be introduced by means of an excess distance beyond the formal convective border (as determined by the Schwarzchild criterion). This distance is defined as $d_{\text {over }}=\alpha_{\mathrm{ov}} H_{\mathrm{p}}$, where $H_{\mathrm{p}}$ is the pressure scale height taken at the edge of the convective core as given by Schwarzschild's criterion and $\alpha_{\mathrm{ov}}$ is a free parameter (see Claret 2007, for a semi-empirical determination of $\alpha_{\mathrm{ov}}$ using double-lined eclipsing binaries). The overshooting is applied only at the core boundary.

2. Opacities. The code uses the tables of opacities provided by Iglesias \& Rogers (1996), completed by the calculations by Alexander \& Ferguson (1994) for lower temperatures.

3. Convection. We adopted the mixing-length theory to describe the envelopes of colder models. Mixing-length is also adopted in the convective cores and can be either adiabatic or superadiabatic.

4. The nuclear network is essentially the same as that described in Claret (2004). The only novelty is the incorporation of measurements of the nuclear reaction ${ }^{14} \mathrm{~N}(\mathrm{p}, \gamma){ }^{15} \mathrm{O}$ (Runkle 2003; Formicola et al. 2004).

5. Numerical details. In the integration of the outer layers, in order to save computational time, we do not assume a fixed luminosity and effective temperature but rather we allow the model to lie within a given area in the HR diagram. In practice, three envelope computations are performed corresponding to a triangle in the HR diagram. To guarantee more accurate computations we decreased the triangle size used in defining an envelope in the HR diagram to $\Delta \log T_{\text {eff }}=0.001$ and $\Delta \log L=0.004$ or smaller. This is particularly important to define the depth of the convective envelope, needed to compute $\lambda_{2}$ (see next section).

The chemical composition of binary systems is not a commonly available observational parameter - which makes the comparison of these systems with theoretical predictions provided by evolutionary models more uncertain - but in the case of $\eta$ And we have two determinations. However, the chemical composition of $\eta$ And is not clear. McWilliam (1990) obtained the first one which is subsolar $([\mathrm{Fe} / \mathrm{H}]=-0.54 \pm 0.25)$, while Taylor (1991) found $[\mathrm{Fe} / \mathrm{H}]=-0.04 \pm 0.11$. To include the observed range of $Z$, we adopted three combinations to model $\eta$ And: $Z=0.006, Z=0.018$ and an intermediate value, $Z=0.012$. These metallicities are scaled to the solar values: $(X, Y, Z)_{\odot}=$ $(0.704,0.279,0.017)$ for the solar mixture by Grevesse \& Sauval (1998).

The first panel of Fig. 1 shows the best fit we found for $\eta$ And. The inferred hydrogen content $\mathrm{X}$ was 0.724 . We have adopted moderate core overshooting, characterized by $\alpha_{\mathrm{ov}}=$ 0.20 that is in good agreement with the determination by Schröder et al. (1997), though those authors used a different chemical composition. The adopted mixing-length parameter $\alpha$ was 1.68 , the same value as we use to calibrate our solar model. We also confirm that the more massive component is the blue loop phase while the secondary is found to be on the giant branch. The derived age is $\log t=8.879_{+0.009}^{-0.007}$. The isochrone that fits the observational data for $\eta$ And is shown more clearly in Fig. 3.

The other two combinations of $Z$ also give acceptable fits, as we can see in the second $(Z=0.018)$ and third $(Z=0.006)$ panel of Fig. 1. In the case of the subsolar metallicity, we have to change the mixing-length ad hoc to 1.45 because the measured effective temperature of the giant star is too low when we 

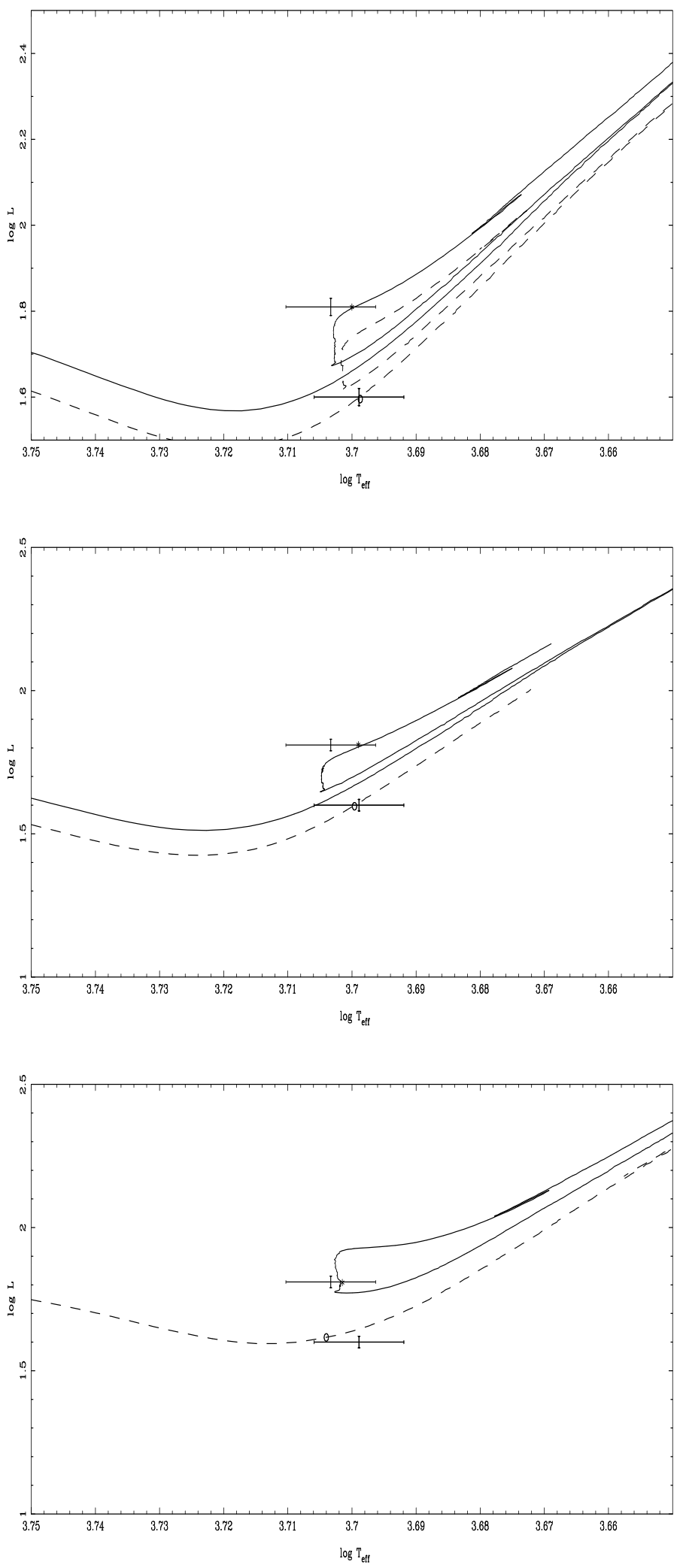

Fig. 1. Theoretical HR diagram and observational parameters for $\eta$ And. The continuous line represents the primary while dashed line denotes the secondary. We have considered moderate core overshooting with $\alpha_{\mathrm{ov}}=0.20$. The luminosity is in solar units and effective temperatures are in $\mathrm{K}$. The asterisk indicates the position of the primary while the open circle denotes the position of the secondary at the ages indicated in the text. The first panel corresponds to the models with $X=0.724$, $Z=0.012$; the second panel to $X=0.706, Z=0.018$ and the third panel $(X=0.742, Z=0.006)$. use the solar mixing-length parameter with a sub-solar composition. The inferred age is $\log t=8.778_{+0.031}^{-0.012}$ which means that it is not as well determined as the previous case. This should be compared with the models computed by Iwamoto \& Saio (1999): these authors found that core overshooting is not necessary to explain the astrophysical parameters of $\eta$ And if models with subsolar metallicity are adopted; their mixing length parameter is very similar to ours (1.40). The approximately solar composition also requires some changes in the input physics. The mixinglength parameter was increased to 1.85 and the final solution is better than that by adopting the models with subsolar metallicity since the inferred age is $\log t=8.918_{+0.003}^{-0.020}$. Comparing our models with $Z=0.018$ with those by Iwamoto \& Saio (1999) (both adopting core overshooting), the mixing-length parameter is similar, as the core overshooting parameter $\left(\alpha_{\mathrm{ov}}=0.20\right.$ and 0.15 , respectively).

Core overshooting is frequently invoked to explain some properties of double-lined eclipsing binaries and colourmagnitude diagrams of clusters. Such an expression is often related to an extra mixing beyond the classical Schwarzchild criterion. Extra mixing produces stellar models with prolonged Main-Sequence times because more fuel is available in the core. In addition, extra mixing also produces effects on later stages of the stellar evolution. A good summary of the global effects of the extra mixing can be found in Schröder et al. (1997). However, other mechanisms may also be acting to increase the core size, for example internal gravity waves or rotation. Talon et al. (1997) investigate the properties of a $9 M_{\odot}$ rotating model, and conclude that the resulting extra mixing is similar to that obtained by adopting moderate core overshooting. We have investigated the dependence of $\alpha_{\mathrm{ov}}$ on stellar mass, i.e. we attributed the extra mixing only to core overshooting, (Claret 2007). One of the conclusions of the study is that core overshooting (or equivalently other mechanisms such as induced mixing by rotation) seems to be necessary to match the absolute dimensions of key double-lined eclipsing binaries as well as the colour-magnitude diagrams of clusters. Keeping this in mind and given the mass range of the systems we are investigating here, we decided to adopt only models with moderate core overshooting. On the other hand, due to the uncertainty in the chemical composition determination of $\eta$ And, we will center our attention on models with an intermediate $Z$, although the conclusions concerning tidal evolution are unaltered.

V2291 Oph is a rare $\zeta$ Aur-type system. The absolute dimensions are given by Griffin et al. (1995) and by Schröder et al. (1996). More or less at the same epoch, Marshal (1996) determined the chemical composition of V2291 Oph. The resulting $[\mathrm{Fe} / \mathrm{H}]$ is $+0.43 \pm 0.2$ and we adopt $Z=0.03$ to characterize the system. Schröder, Pols \& Eggleton (1997) analysed V2291 Oph and found an acceptable fit by adopting $Z=0.02$ and a moderate amount of core overshooting $\alpha_{\mathrm{ov}} \approx 0.27$. Iwamoto \& Saio (1999), on the other hand, using the same astrophysical parameters but adopting a different chemical composition ( $Z=0.03$ according to Marshal 1996), also found a good match. In both cases, a larger value of the mixing-length parameter was adopted (2.0 and 2.3, respectively). We have adopted $(X, Z)=(0.64,0.03), \alpha=2.5$ and $\alpha_{\mathrm{ov}}=0.20$. The comparison is shown in the first panel of Fig. 2. The position of the secondary (less massive component) is more difficult to fit and defines the age error bars. The inferred age is $\log t=8.271_{+0.090}^{-0.020}$.

SZ Cen is a double-lined eclipsing binary whose primary has evolved beyond the TAMS while the secondary is still in the Main-Sequence (Andersen 1975, 1991) As far as we know, there are no chemical observations for SZ Cen. The models which fit 

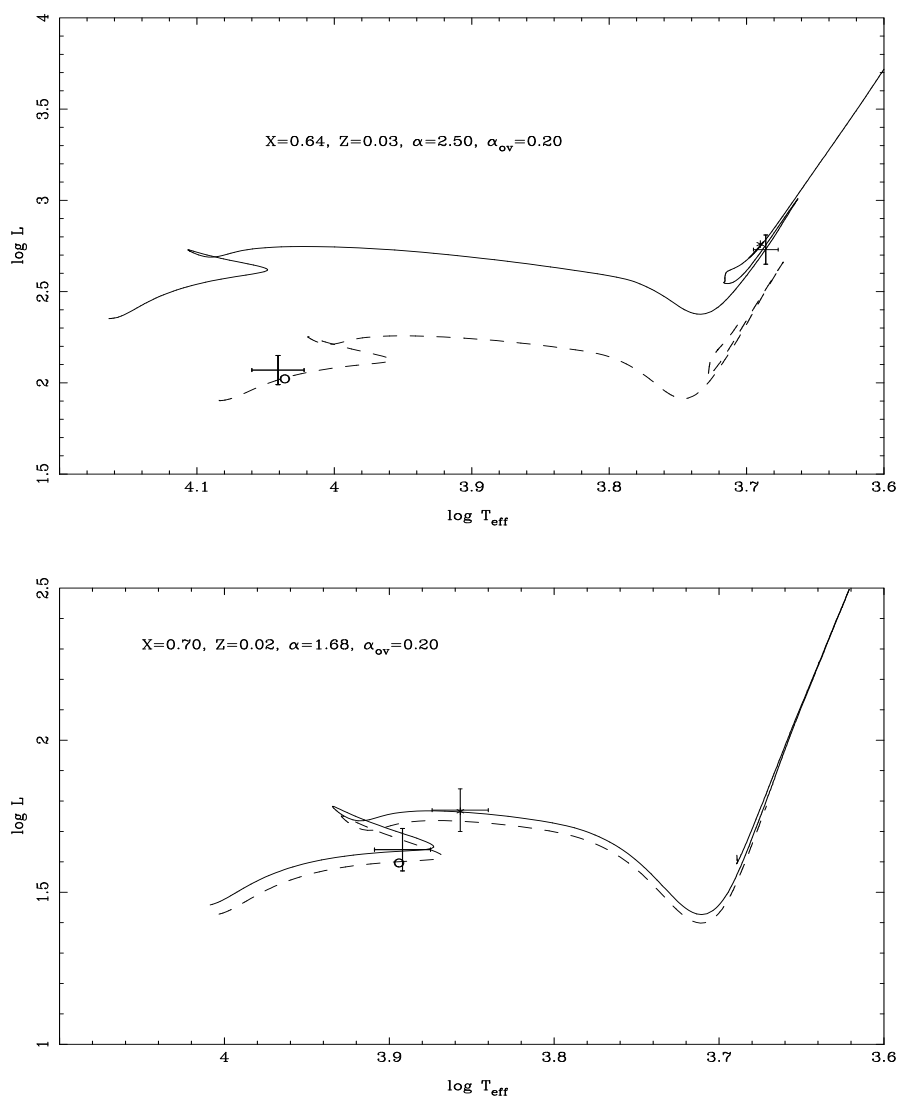

Fig. 2. Theoretical HR diagram and observational parameters for V2291 Oph (first panel), and SZ Cen (second panel). Same symbols as in Fig. 1.

its absolute dimensions better are those with moderate core overshooting and $(X, Z)=(0.70,0.02)$, (see second panel of Fig. 2$)$. The derived age is $\log t=8.869_{+0.001}^{-0.014}$.

\section{Equations of tidal evolution}

In binary systems, the viscous dissipation of time-dependent tides acts to synchronize the stellar rotation and circularize the orbit. As a consequence of tidal interactions we expect either a coalescence due to the spiralling of both stars or an equilibrium situation characterized by a coplanar circular orbit and with both rotational angular velocities synchronized with the orbital period $P$. The explicit equations of tidal evolution are complicated and they are coupled. The derivative $\mathrm{d} e / \mathrm{d} t$ depends on the internal structure of the stars, on the eccentricity as well as on the ratio $\Omega_{i} / \Omega_{\mathrm{o}}$ where $\Omega_{i}$ is the angular velocity of the star $i$ and $\Omega_{\mathrm{o}}$ is the mean orbital angular velocity. On the other hand, the derivative $\mathrm{d} \Omega_{i} / \mathrm{d} t$ also depends on the same parameters. Such differential equations can be integrated if we know the initial conditions. Unfortunately this is not the case. In order to obtain some information on the tidal evolution of binary stars, some simplifications are assumed in the mentioned equations: small eccentricities and small departure of synchronism which lead to the time scales of circularization/synchronization (Eqs. (1)-(9)). In this way, only relative values of the eccentricity and rotational angular velocity can be obtained. The integrations are carried out until the relative variations of eccentricity and angular velocities become 0.5 per cent of the original values (for more details, see Zahn 1977, 1989). The evolutionary ages at which this occurs are the critical time $t_{\mathrm{cir}}$ and $t_{\mathrm{sync} 1,2}$, respectively. The mentioned simplifications limit our conclusions concerning tidal evolution, specially for those systems showing high values of eccentricities and/or high levels of asynchronism. As mentioned above, a better approach to the tidal evolution problem consist of integrating the complete set of coupled differential equations simultaneously with the stellar evolution equations, as done for example by Eggleton \& Kiseleva-Eggleton (2002) for cool Algols systems, or more recently by Stancliffe \& Eldridge (2009) for the evolution of the progenitor of Supernova 1993J. However, the combination of initial trial parameters (period, rotational velocities and eccentricity) is large and a more detailed treatment is needed. At present we are involved in a new code version which takes into account such combinations, as well as a Monte Carlo error analysis to estimate the uncertainties in the circularization/synchronization levels (Torres et al. 2009). However, such calculations are beyond the scope of the present paper. We hope to present the results in the near future by using double-lined eclipsing binaries with more accurate absolute dimensions that are suitable to test the current tidal evolution theories.

The simplified differential equations that describe the variations of the eccentricity and the angular rotational velocities in a binary system are

$\frac{\mathrm{d} \ln \left(\Omega_{i}-\Omega_{\mathrm{o}}\right)_{k}}{\mathrm{~d} t}=-\frac{1}{\tau_{\mathrm{syn}, k}}$

where $\Omega_{0}$ and $\Omega_{i}$ were already defined, $k=1,2$ refer to the primary and secondary component, respectively and $\tau_{\mathrm{syn}, k}$ is the time scale of synchronization and

$\frac{\mathrm{d} \ln e}{\mathrm{~d} t}=-\frac{1}{\tau_{\mathrm{cir}}}$

where $e$ is the eccentricity and $\tau_{\text {cir }}$ is time scale of circularization. To integrate the above differential equation we have to consider the contribution of both components

$\frac{1}{\tau_{\mathrm{cir}}}=\frac{1}{\tau_{\mathrm{cir}, 1}}+\frac{1}{\tau_{\mathrm{cir}, 2}}$

where subscripts 1 and 2 refer to the primary and the secondary component.

There are three mechanisms which have been invoked to explain the orbital evolution of binary systems. The first braking mechanism is due to Tassoul $(1987,1988)$. Following this description, the stars in a binary system tend to synchronize and circularize the orbit due to the tidal distortions which cause large scale hydrodynamical currents. Following Tassoul: "The hydrodynamical mechanism involves a large scale meriodional flow, superposed on the motion around the rotation axis of the tidally distorted component. These transients (due to the lack of axial symmetry), mechanically driven currents - which are much faster than the steady, termally driven Eddington-Vogt currents - cease to exist as soon as synchronization has been achieved in the star". On the other hand, in a binary system, a star is subject to the gravitational field of its companion and from this interaction a tidal bulge appears. If we assume that both stars are synchronized, this bulge is aligned but if there is departure from synchronism a delay gives rise to a torque. This torque tends to bring the star back into the synchronism. Zahn $(1975,1989)$ indentified two mechanisms: turbulent dissipation and radiative damping. Both mechanisms are responsible for the tidal friction on late-type and early-type stars, respectively. For a more extensive discussion on the three mechanisms see Claret \& Cunha (1997). 
For the hydrodynamical mechanism we compute the time scales for circularization and synchronization following Tassoul (1988):

$\tau_{\mathrm{Hsyn}}=5.35 \times 10^{2+\gamma-N / 4} \frac{(1+q)}{q} L^{-1 / 4} M^{5 / 4} R^{-3} P^{11 / 4}$

$\tau_{\text {Hcir }}=9.4 \times 10^{3+\gamma-N / 4}(1+q)^{2 / 3} \beta^{-2} L^{-0.25} M^{23 / 12} R^{-5} P^{49 / 12}$

where $q$ is the mass ratio, $\beta$ the radius of gyration, $P$ the orbital period in days, and $M, L$ and $R$ are the stellar mass, luminosity and radius in solar units. The parameter $N$ is related to the different ways to transport energy into the outer layers of the stars in such way that $10^{N}=v_{t} / v_{r}$, where $v_{t}$ and $v_{r}$ are the eddy and radiative viscosities, respectively. For stars with envelopes in convective equilibrium, it is often assumed that $N=10$, while $N=0$ is assumed for stars whose envelopes are in radiative equilibrium. The adjustable parameter $\gamma$ was introduced by Tassoul in order to take into account some limitations inherent to the time scale derivation, for example, that the spin-down times is only a lower limit. It was calibrated by Claret et al. (1995) who obtained $\gamma=1.6$ by using double-lined eclipsing binaries.

For the case of turbulent dissipation (convective envelopes):

$\tau_{\mathrm{TDsync}}=3.95 \times 10^{2} \beta^{2} M^{7 / 3} \frac{(1+q)^{2}}{q^{2}} L^{-1 / 3} \lambda_{2}^{-1} \frac{P^{4}}{R^{16 / 3}}$

$\tau_{\mathrm{TD} c i r c}=1.99 \times 10^{3} M^{3} \frac{(1+q)^{5 / 3}}{q} L^{-1 / 3} \lambda_{2}{ }^{-1} \frac{P^{16 / 3}}{R^{22 / 3}}$.

For stars with convective cores and radiative envelopes (radiative damping) the time scales are:

$\tau_{\mathrm{RDsync}}=2.03 \beta^{2} M^{7 / 3} \frac{(1+q)^{2}}{q^{2}} E_{2}{ }^{-1} \frac{P^{17 / 3}}{R^{7}}$

$\tau_{\text {RDcirc }}=1.71 \times 10^{1} M^{3} \frac{(1+q)^{5 / 3}}{q} E_{2}^{-1} \frac{P^{7}}{R^{9}}$.

In these equations $M, R$ and $L$ are in solar units while $P$ is given in days and the time scales are given in years. The torque constant $E_{2}$ is related to the dynamic tidal contribution to the total perturbed potential and it is similar to $k_{2}$ (the apsidal motion constant) but it is much more dependent on the stellar structure than the mentioned constant. Concerning the calculation of $\lambda_{2}$, that is connected to the structure of the external layers, we refer the readers to Claret \& Cunha (1997). The differential Eqs. (1)-(2) were integrated for each system (using the time scales given by Eqs. (4)-(9)) through a fourth order Runge-Kutta method. The radius of gyration and the torque constant as well as other astrophysical parameters such as the age, radius, luminosity, etc are directly inferred from the same evolutionary stellar models used to compare the observed properties of $\eta$ And, V2291 Oph and SZ Cen.

\section{The tidal evolution of $\eta$ And, V2291 Oph and SZ Cen; final remarks}

\section{1. $\eta$ And}

The orbital elements of $\eta$ And present some ambiguities, for example, the derived eccentricity. Hummel et al. (1993) give the value $0.006 \pm 0.002$ while Pourbaix et al. (2004) found $e=0.0081$ and Massarotti et al. (2008) derived the value
$0.0032 \pm 0.0044$. The most recent determination of $e$ was provided by Griffin (private communication, $0.003 \pm 0.0020$ ). Given that value and the error bars, we can conclude that the orbit is practically circular and this imposes a constraint on the calculations of the critical times of circularization through Eqs. (2), (3), (5), (7) and (9).

Massarotti et al. (2008) obtained the projected rotational velocities for both components of $\eta$ And: $V_{\text {rotA }} \sin i=4.6 \mathrm{~km} \mathrm{~s}^{-1}$ and $V_{\text {rotB }} \sin i=4.7 \mathrm{~km} \mathrm{~s}^{-1}$. Hummel et al. (1993) derived the inclination of the orbit, $i \approx 30^{\circ}$, which gives for both components $\approx 9 \mathrm{~km} \mathrm{~s}^{-1}$. The theoretical values are, respectively, $V_{\text {rotAT }}=$ $4.6 \mathrm{~km} \mathrm{~s}^{-1}$ and $V_{\text {rotBT }}=3.7 \mathrm{~km} \mathrm{~s}^{-1}$. Within the uncertainties, we can consider that both components are not synchronized with the orbital period.

Considering such data and integrating the above differential equations, we are finally able to analyse the tidal evolution of $\eta$ And. Note that during the integration we assume that the orbital period is constant since the rotational angular momentum of both stars is much smaller than the orbital one. While on the main sequence, $\eta$ And and V2291 Oph have convective cores and radiative envelopes. However, as they evolve to giants, core convection shuts down and a convective envelope develops. This has the effect of changing which tidal mechanism is important: for the hydrodynamical mechanism, we will have a transition in $N$ which is related to the different mechanisms of transport of energy in the outer layers. Equally, we have a transition between the radiative damping and turbulent dissipation for the same reasons explained above. For practical purpose, we prefer to use the effective temperature as a criterion, instead of masses, to select the best value of $N$.

We select the model with $(X, Z)=(0.724,0.012)$ and $\log t=$ $8.879_{+0.009}^{-0.007}$ to investigate the tidal evolution of $\eta$ And. The hydrodynamical mechanism predicts that the orbit of $\eta$ And should be circular (Fig. 3, first panel) since its critical time for circularization is $\log t_{\mathrm{cir}}=8.806$, which is smaller than the inferred age of the system considering its error bars; therefore it is compatible with the observed eccentricity. Theoretical calculations predict that the primary component should be synchronized $\left(\log t_{\text {sync1 }}=8.800\right)$ which is in contradiction to observations. On the other hand, the prediction for the secondary is $\log t_{\text {sync2 } 2}=8.864$, which is shorter than the inferred age, and this is also in disagreement with observations.

The radiative damping and turbulent dissipation are analysed using the same stellar models and the main results are shown in Fig. 3 (second panel). Following these mechanisms, the orbit should be circular; the critical time for circularization is $\log t_{\text {cir }}=8.872$ which is shorter than the inferred age. This is in agreement with the observed level of circularization. The situation of synchronization is similar to that predicted by the hydrodynamical mechanism: the primary achieves synchronization near the tip of the red giant branch $\left(\log t_{\text {sync } 1}=8.805\right)$ while the secondary achieves the equilibrium state at the same phase at $\log t_{\text {sync2 }}=8.870$. As previously commented, both components of $\eta$ And are still not synchronized and the theoretical predictions are in clear disagreement with these data. Concerning synchronization, some words of caution should be given. Unlike the case of circularization, where the eccentricity is more clearly established, the analysis of the rotational velocities is not so straightforward. As is known, the observed rotational velocities correspond to the upper layers of the stars. However, there is a possibility that these are decoupled from the rotational velocities in the core.

As we have previously seen, the critical time of synchronization for the primary indicates that this would be synchronized 

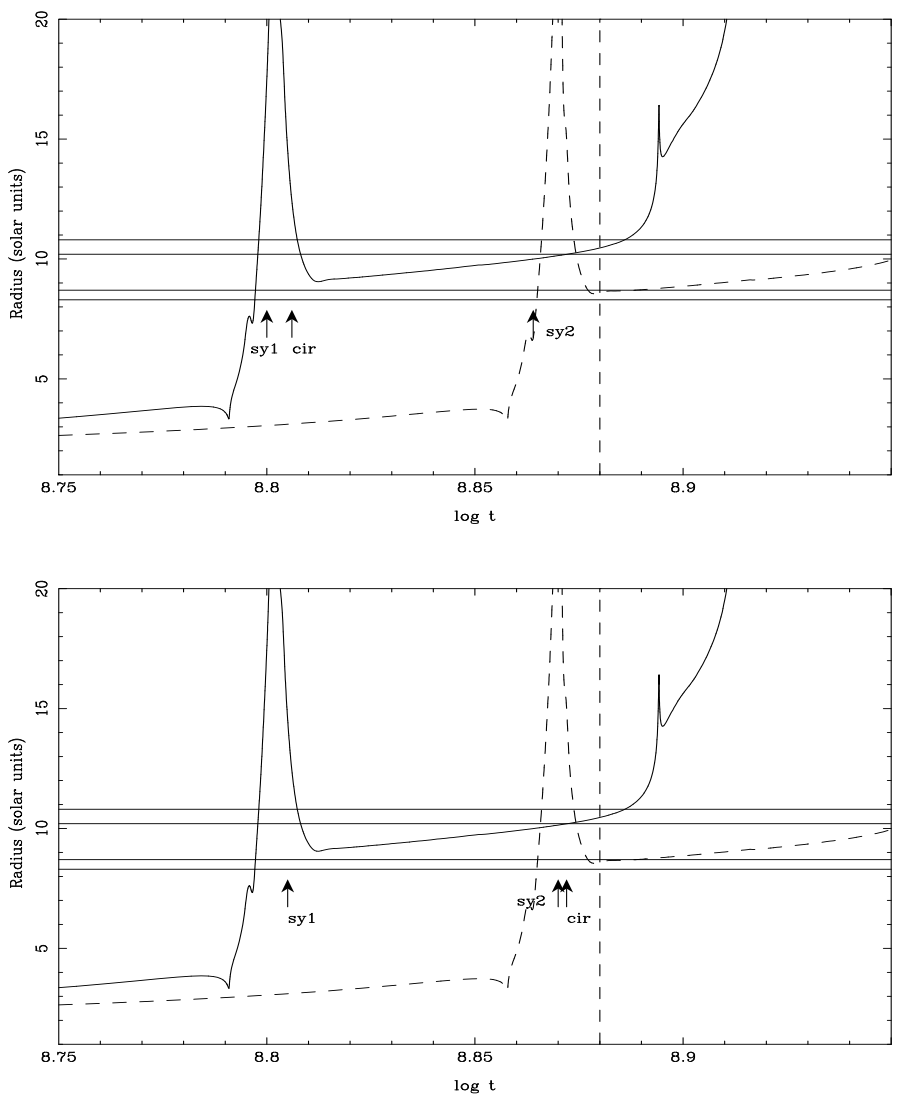

Fig. 3. Radius evolution of $\eta$ And (continuous line represents the primary and the dashed one the secondary). The radius is in solar units while the time is given in years. The horizontal lines denote the observed radii and their error bars and the arrows indicate the times for circularization (circ), for synchronization of the primary (sy1) and secondary (sy2). The vertical dashed line marks the inferred age of the system. First panel: hydrodynamical mechanism; second panel: turbulent dissipation and radiative damping mechanisms.

near the tip of the giant branch. However, from Fig. 3, we note that it immediately contracts. Assuming that it rotates as a rigid body (see Fig. 4) we can estimate the spin-up due to contraction: around 4 times faster than it achieves the tip of the giant branch. This might be a possible explanation for the disagreement between the observed rotational velocity and the critical time of synchronization.

Although there are uncertainties in the rotational velocities of the components of $\eta$ And, they can be used to infer, in a first approximation, the angular momentum distribution within the stars. If we assume that both stars rotate as rigid bodies, we can predict the rotation evolution with respect to the ZAMS velocities. In spite of the uncertainty in $\sin i$ or in the intrinsic values of the rotational velocities, the observed ratio $V_{\text {rotA }} / V_{\text {rotB }}$ is unaltered by these sources of error and can be followed as a function of time. We show such an evolution in Fig. 4. The observed ratio $\approx 1.0$ and the theoretical $V_{\text {rotA }} / V_{\text {rotB }}$ we derive at the inferred age of the system in Fig. 4 is 1.09. This should be compared with the value obtained for TZ For (Claret \& Giménez 1995) for which an observed ratio of 10 was measured, also in good agreement with the theoretical value.

\subsection{V2291 Oph}

One of the most serious limitations of V2291 Oph concerning its tidal evolution is the evolutionary time scales of both

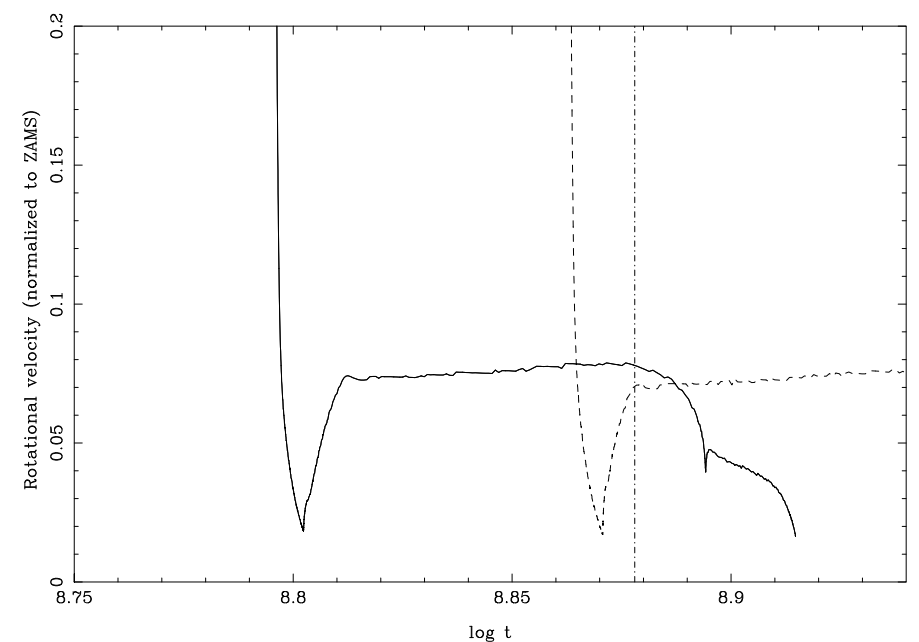

Fig. 4. The rotational evolution of $\eta$ And. The primary is represented by the continuous line and the secondary by the dashed one. The inferred age of the system is marked with a vertical dashed-dotted line.

components. The mass of the primary is about $30 \%$ higher that the secondary and the time scales are so different that a direct integration of the differential equations is not possible, mainly in the case of the turbulent dissipation/radiative damping mechanisms. This is because of the condition assuring that the system is synchronized (or circularized) - relative variations of eccentricity and angular velocities became 0.5 per cent of the original values - cannot be fulfilled for both time scales of V2291 Oph simultaneously (even if we allow a less restrictive condition). As is well known, the hydrodynamical mechanism is more efficient than turbulent dissipation and radiative damping. Therefore, we present here only the calculations made by adopting the hydrodynamical mechanism. On the other hand, no information is available on the rotational velocities. Of course, no substantial amount of information can be extracted from the study of this system. However, even without the observed rotational velocities, we can predict the synchronization time for both components to be compared with future observations.

The results by adopting the hydrodynamical mechanism are shown in Fig. 5. The critical times of synchronization of both components is predicted to be near the tip of the giant branch. On the other hand, the orbit of V2291 Oph is highly eccentric $(e \approx 0.31)$ and the critical time for circularization $\left(\log t_{\mathrm{cir}}=\right.$ $8.277)$ is slightly longer than the inferred age $(\log t=8.271)$ but well within the error bars for the age. Although this critical time is compatible with the observed eccentricity, due to the high value of $e$ and due to the small difference between the critical time for circularization and age one would expect that this difference is not enough to circularize the orbit. The high eccentricity of V2291 Oph leads to an interesting question: did this system originally begin with a very high initial eccentricity (say, 0.8 ) that has been reduced to 0.3 ? Or maybe that the eccentricity was initially near 0.30 and has not undergone strong tidal forces? As the system shows a long orbital period, the corresponding tidal forces are relatively weak and the latter option seems to be the most probable. However, the above analysis should be taken with caution. As mentioned in the beginning of Sect. 3, the high value of the present eccentricity is clearly not consistent with the adopted assumption used to derive the time scale of circularization. More complete and accurate data may shed some light on this ambiguous situation although they will be not enough to remove the mentioned inconsistency. We encourage observers to 


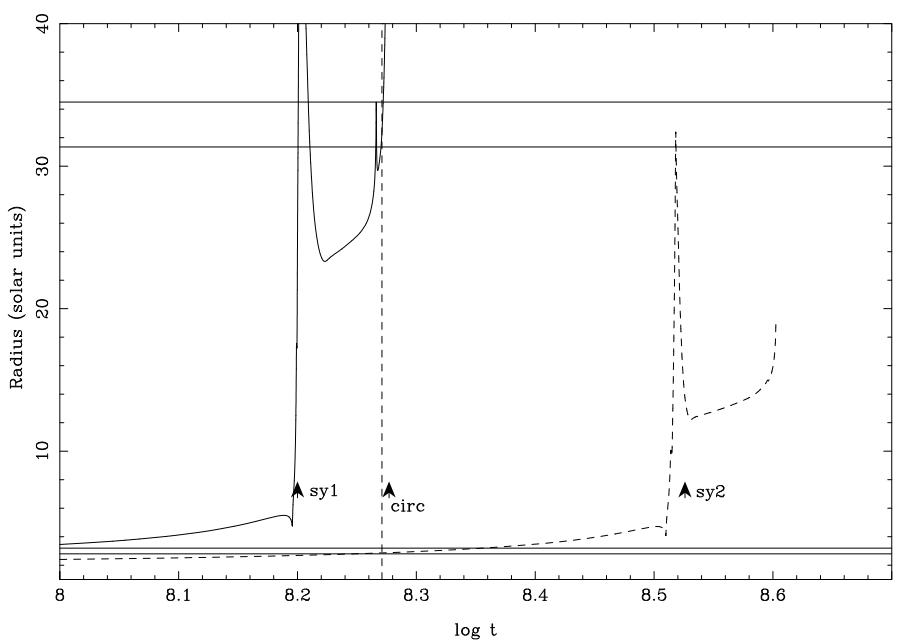

Fig. 5. The same as in Fig. 3 but for V2291 Oph. Hydrodynamical mechanism.
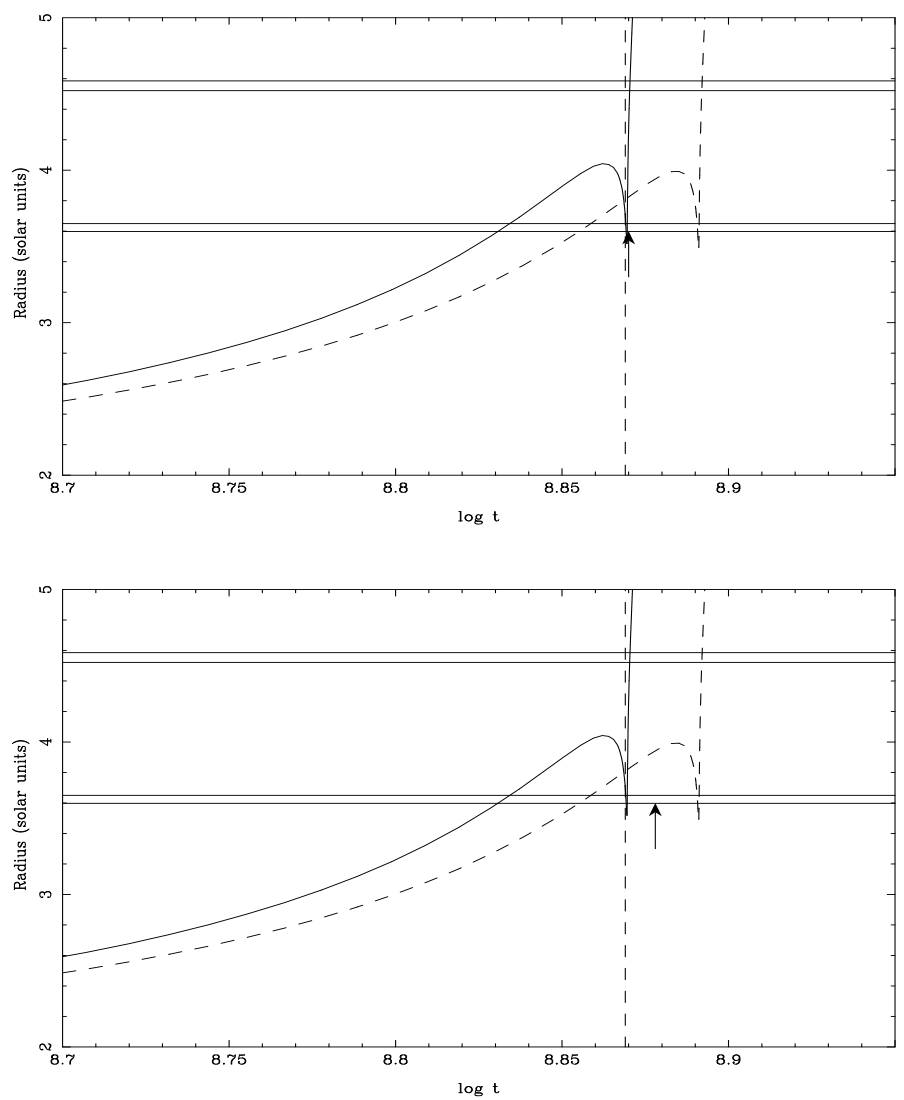

Fig. 6. The same as in Fig. 3 but for SZ Cen. Hydrodynamical mechanism and turbulent dissipation/radiative damping. The critical times for synchronization for the hydrodynamical mechanism are not shown due to the difference in time scale. Because the critical times of circularization and synchronization are very similar, only one arrow is show for the radiative damping mechanism.

improve and complete the astrophysical data for V2291 Oph and other similar systems. The benefits are worth the effort since we have good information of only one system showing very evolved components (TZ For).

\subsection{SZ Cen}

SZ Cen presents a circular orbit and the observed values of the rotation velocities are $60 \pm 5$ and $44 \pm 4 \mathrm{~km} \mathrm{~s}^{-1}$ for the primary and secondary, respectively. The theoretical rotational velocities at the periastron are 56 and $45 \mathrm{~km} \mathrm{~s}^{-1}$ and we can consider both components as synchronized. The calculations made by adopting the hydrodynamical mechanism reveal that the critical time for circularization is $\log t_{\text {cir }}=8.740$, that is smaller than the inferred age $(\log t=8.869)$. Such a result is compatible with the circular orbit of SZ Cen. Concerning the levels of synchronization, the hydrodynamical mechanism seems to be able to explain the rotational velocities since the critical times are about 2 order of magnitude shorter than the inferred age $\left(\log t_{\text {sync1,2 }} \approx 6.2\right)$.

On the other hand, the radiative damping mechanism predicts a critical time for circularization longer that the age of the system $\left(\log t_{\mathrm{cir}}=8.878\right)$ and therefore it is not compatible with the circular orbit of SZ Cen. Following this mechanism, the primary should be synchronized at $\log t_{\text {sync1 }}=8.877$, more or less at the same time as the system circularizes. The secondary will synchronize the orbit a little later, at $\log t_{\text {sync } 1}=8.890$. This seems to be in disagreement with the observed rotational velocities since they indicate that both components are synchronized. However, given the small differences between the critical times for synchronization, and the inferred age, the associated stellar models, the tidal theories and the observational data may be not accurate enough to elucidate the question. On the other hand, by applying the same simple method to follow the rotational evolution as described in the case of $\eta$ And, we have found that the predicted $V_{\text {rotA }} / V_{\text {rotB }}$ is around 1.2 while the observed value is 1.3 .

Although the actual theories of tidal braking are able to explain some tidal characteristics of our three systems, some predictions are in disagreement with the observed eccentricities and rotational velocities. In addition, we should remember that there are also problems of modelling double-lined binary systems with very evolved components, such as the unique case of TZ For. Only for a high value of $\alpha_{\text {ov }}$ was it possible to place both components in the same isochrone (Claret 2007). This may be an evidence that there are problems with the late stellar evolutionary stages. It is clear that we need to obtain more accurate data for binaries with very evolved components to elucidate such discrepancies and ambiguities.

Acknowledgements. I thank R. Griffin for providing up-to-date data of $\eta$ And. The Spanish MEC (AYA2006-06375) is gratefully acknowledged for its support during the development of this work.

\section{References}

Andersen, J. 1975, A\&A, 45, 203

Andersen, J. 1991, A\&AR, 3, 91

Alexander, D. R., \& Ferguson, J. W. 1994, ApJ, 437, 879

Campbell, W. W.,\& Wright, W. H. 1900, ApJ, 12, 256

Claret, A. 2004, A\&A, 424, 919

Claret, A. 2005, in Tidal Evolution and Oscillations in Binary Stars, ed. A. Claret, A. Giménez, \& J.-P. Zahn, ASP Conf. Ser., 333, 122

Claret, A. 2007, A\&A, 475, 1019

Claret, A., \& Cunha, N. C. S. 1997, A\&A, 318, 187

Claret, A., \& Giménez, A. 1995, A\&A, 296, 180

Claret, A., Giménez, A., \& Cunha, N. C. S. 1995, A\&A, 299, 724

Eggleton, P. P., \& Kiseleva-Eggleton, L. 2002, ApJ, 575, 461

Formicola, A., Imbriani, G., Costantini, H., et al. 2004, Physics Letters B, 591, 61

Gordon, K. C. 1946, ApJ, 103, 13

Grevesse, N., \& Sauval, A. J. 1998, Space Sci. Rev., 85, 161

Griffin, R. E. M., Marshall, K. P., Griffin, R. F., \& Schr̈oder, K.-.P. 1995, A\&A, 301,217 
Hummel, C. A., Armstrong, J. T., Quirrenbach, et al. 1993, AJ, 106, 2486

Habets, G. M. H. J., \& Zwaan, C. 1989, A\&A, 211, 56

Iglesias, C. A., \& Rogers, F. J. 1996, ApJ, 464, 943

Iwamoto, N., \& Saio, H. 1999, ApJ, 521, 297

Marshall, K. P. 1996, MNRAS, 280, 977

Massarotti, A., Latham, D. W. Stefanik, R. P., \& Fogel, J. 2008, AJ, 135, 209

Mayor, M., \& Mermilliod, J. C. 1984, in Observational tests of the stellar evolution theory, ed. A. Maeder, \& A. Renzini, (Dordretch: D. Reidel Publ. Co.), 411

McWilliam, A. 1990, ApJS, 74, 1075

Meibom, S., \& Mathieu, R. 2005, in Tidal Evolution and Oscillations in Binary

Stars, ed. A. Claret, A. Giménez, \& J.-P. Zahn, ASP Conf. Ser., 333, 95

Pourbaix, D., Tokovinin, A. A., Batter, A. H., et al. 2004, A\&A, 424, 727
Runkle, R. C. 2003, Ph.D. Thesis, University of North Carolina, unpublished Schröder, K.-P., Marshall, K. P., \& Griffin, R. E. M. 1996, A\&A, 311, 631 Schröder, K.-P., Pols, O. R., \& Eggleton, P. P. 1997, MNRAS, 285, 696 Stancliffe, R. J., \& Eldridge, J. J. 2009, MNRAS, 396, 1699

Talon, S., Zahn, J.P., Maeder, A., \& Meynet, G. 1997, A\&A, 322, 209 Taylor, B. J. 1991, ApJS, 76, 715

Tassoul, J. L. 1987, ApJ, 322, 856

Tassoul, J. L. 1988, ApJ, 324, L71

Torres, G., Lacy, C. H. S., \& Claret, A. 2009, AJ, in press

Zahn, J. P. 1975, A\&A, 41, 329

Zahn, J. P. 1977, A\&A, 57, 383

Zahn, J. P. 1989, A\&A, 220, 112 